\title{
ORIGINAL ARTICLE \\ Reference values for the transformed Van Lieshout hand function test for tetraplegia
}

\author{
AIF Spooren ${ }^{1,2}$, C Arnould ${ }^{3}$, RJEM Smeets ${ }^{1,2}$, G Snoek ${ }^{4}$ and HAM Seelen ${ }^{1,2}$
}

Study design: Longitudinal cohort study.

Objectives: Previously, the Van Lieshout hand function test for tetraplegia (short form)(VLT-SF) has been transformed into an interval scale (r_VLT-SF) using the Rasch analysis, thereby fulfilling the requirements of an objective measurement. The present study aims (1) to transform r_VLT-SF $F_{\text {logit }}$ Scores $\left(r_{-}\right.$VLT-SF $\left.F_{\text {logit }}\right)$ into 0-100 scores $\left(r_{-} V L T-S F_{100}\right)$ in order to facilitate communication amongst its users and (2) to describe r_VLT-SF 100 reference values in order to refine the prediction of upper extremity skilled performance in persons with tetraplegia.

Setting: Eight Dutch rehabilitation centres.

Methods: The VLT-SF data of tetraplegic patients from eight Dutch rehabilitation centres, gathered at the start of the active rehabilitation (t1), 3 months after $\mathrm{t} 1$ ( $\mathrm{t} 2$ ) and at the time of discharge (t3), have been used. The $\mathrm{r}_{\text {_VLT-SF}}$ 100 total score for $\mathrm{t} 1$ and $\mathrm{t} 3$ were computed, anchored on the data for $\mathrm{t} 2$. Reference values (medians and interquartiles) for different subgroups were calculated at different moments during the rehabilitation.

Results: Data at t1 $(n=64)$, t2 $(n=73)$ and $\mathrm{t} 3(n=69)$ were used. The r_VLT-SF ${ }_{\text {logit }}$ scores $(-4.78-6.32)$ were transformed into r_VLT-SF $100(0-100)$ at t2. Reference values were established for persons with motor complete or incomplete lesions with either a high (C1-C6) or a low (C7-T1) lesion level at t1, t2 and t3. Significant differences $(P<0.025)$ in $r_{-} V L T-S F_{100}$ were found for most subgroups and different time intervals.

Conclusions: The r_VLT-SF $F_{\text {logit }}$ scores were converted into $r_{-} V L T-S F_{100}$ Scores, and reference values of the $r_{-} V L T-S F_{100}$ have been established for different subgroups of tetraplegic patients at different stages during rehabilitation. The r_VLT-SF 100 has been proven to be able to detect improvement over time.

Spinal Cord (2013) 51, 745-749; doi:10.1038/sc.2013.73; published online 30 July 2013

Keywords: spinal cord injury; upper extremity; reference values; test; motor skill

\section{INTRODUCTION}

The Van Lieshout hand function test for tetraplegia (VLT) is an instrument to assess the quality of arm-hand skilled performance (AHSP) in persons with a cervical spinal cord injury (C-SCI) and has been demonstrated to be a valid, reliable and responsive measure. ${ }^{1-3}$ It is used not only as an assessment tool for upper extremity functioning but also as guidance for the therapy management regarding arm and hand performance (http://www.vlt-test.eu). Because the original VLT features an ordinal scale that does not satisfy the requirements of objective measurement, a Rasch analysis of the VLT has been performed. ${ }^{4}$ After recoding the response categories of the original short form VLT (VLT-SF), VLT-SF has been transformed into an interval scale (r_VLT-SF), using the Rasch analysis. It was found that the r_VLT-SF satisfied the criterion of unidimensionality. This means that the r_VLT-SF data fitted the model. This model prescribes that only the person's ability, the item difficulty and the threshold difficulty determine the probability of endorsing a category for an item. ${ }^{5,6}$

An interval scale enables clinicians to better quantify the individual progress, to compare the outcome of patients between each other and to derive expected items scores. ${ }^{4}$
The total score of the $r_{-}$VLT-SF is expressed in so-called logits $\left(r_{-}\right.$VLT-SF $F_{\text {logit }}$ ), which are defined as the probability units that express the natural logarithm of the odds of success (that is, the pass-to-fail ratio of a subject to an item). ${ }^{7}$ However, clinicians and patients are not used to the concept of logits. They rather prefer to use scores that are more meaningful to them. The use of scores ranging from 0 to 100 instead of scores in logits (in case of $r_{-}$VLT-SF $F_{\text {logit }}$ ranging from -4.78 to 6.32) would enhance the communication, not only amongst therapists, but also between patients and therapists. Therapists would be able to communicate with patients in terms of 'You have nearly reached the maximal score of 100' or 'You progressed from a score of 60 to 80 ', rather than 'You progressed from 1.81 to 3.88'. Spooren et al. ${ }^{4}$ reported that the r_VLT-SF $F_{\text {logit }}$ allows the derivation of the expected VLT-SF item scores and identification of the VLT-SF item at which the patient is lagging behind. However, it would also be beneficial to be able to predict the outcome of arm and hand treatment in terms of 'What will the patient eventually be able to do with his arms and hands?' The establishment of the reference values of the VLT-SF for different patient groups at different time points during rehabilitation may help to improve the prediction of the outcome regarding AHSP.

${ }^{1}$ Rehabilitation Sciences Adelante Centre of Expertise in Rehabilitation and Audiology, Hoensbroek, The Netherlands; ${ }^{2}$ Department of Rehabilitation Medicine, Research School CAPHRI, Maastricht University, Maastricht, The Netherlands; ${ }^{3}$ Departments of Paramedical Category, Physical and Occupational Therapy, Haute Ecole Louvain en Hainaut, Montignies-sur-Sambre, Belgium and ${ }^{4}$ Roessingh Rehabilitation Centre, Enschede, The Netherlands

Correspondence: Dr AIF Spooren, Rehabilitation Sciences Adelante Centre of Expertise in Rehabilitation and Audiology, Zandbergsweg 111, Hoensbroek 6432 CC, The Netherlands.

E-mail: a.spooren@adelante-zorggroep.nl

Received 6 July 2012; revised 18 June 2013; accepted 20 June 2013; published online 30 July 2013 
The aim of the present study was (1) to convert $r_{-}$VLT-SF $F_{\text {logit }}$ into r_VLT-SF $100 ;$ (2) to establish reference values of $r_{-}$VLT-SF ${ }_{100}$ for different groups of patients at different moments during rehabilitation and (3) to verify whether the r_VLT-SF 100 is able to detect changes over time in different subgroups.

\section{METHODS}

Data of patients collected on behalf of the umbrella project 'Physical Strain, Work Capacity and Mechanisms of Restoration of Mobility in the Rehabilitation of Persons with SCI' (http://www.scionn.nl) across eight Dutch rehabilitation centres and of the ToCUEST project (in Adelante Rehab Centre) were used. ${ }^{8,9}$ The inclusion criteria were (in)complete C-SCI (including lesion at the motor level T1 according to the International Standard for Neurological Classification of Spinal Cord Injury (ISNCSCI) ${ }^{10}$ ) and being between 18 and 70 years of age. The exclusion criteria were severe additional neurological, orthopaedic or rheumatologic diseases hampering AHSP and total inability to perform upper extremity measurements. Research assistants experienced in performing the VLT-SF assessment collected data on the best hand. The VLTSF consists of 10 items with regard to arm positioning and stabilising, hand opening and closing, grasping and releasing and manipulating (http://www.vlttest.eu). For the current study, data at three moments during the rehabilitation were used; that is: at the start of the active rehabilitation (t1) (when the patient is able to sit for at least 3 consecutive hours), 3 months after $\mathrm{t} 1$ (t2) and at the time of discharge ( $\mathrm{t} 3)$.

Because lesion completeness and lesion level mainly determine the level of arm and hand functioning, ${ }^{11}$ reference values are reported for motor lesion completeness (that is, for Grade A or B on the ASIA (American Spinal Injury Association) Impairment Scale (AIS) ${ }^{10}$ and for Grade C or D on AIS) and lesion level (that is, for high (C1-C6) or low (C7-T1) lesion level according to the ISNCSCI). ${ }^{10}$ Furthermore, as the outcome of AHSP improves across different stages of rehabilitation, reference values from different moments of time (that is, $\mathrm{t} 1, \mathrm{t} 2$ and $\mathrm{t} 3$ ) are reported.

\section{Data analysis}

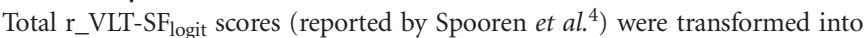
total $r_{-}$VLT-SF 100 scores for the t 2 time moment (that is, 3 months after the start of the active rehabilitation). As theories on the Rasch analysis indicate that one data set should be used as the basic set on which the data sets of other measurement moments should be anchored, ${ }^{5}$ total $\mathrm{r}_{-} \mathrm{VLT}_{-} \mathrm{SF}_{100}$ scores of $\mathrm{t} 1$ and $\mathrm{t} 3$ were calculated anchored on the $\mathrm{r}_{-}$VLT-SF 100 at $\mathrm{t} 2$ (that is, the $\mathrm{r}_{-}$VLT$\mathrm{SF}_{100}$ scores at $\mathrm{t} 1$ and $\mathrm{t} 3$ were derived based on the $\mathrm{r}_{-}$VLT-SF 100 scores at $\mathrm{t} 2$ ).

Based on the $r_{-}$VLT-SF $F_{100}$ scores at $t 1, t 2$ and $t 3$, reference values were established using the median and interquartiles for the different subgroups; that is, persons with motor complete and incomplete lesions followed by a division into persons with a high (C1-C6) and a low (C7-T1) lesion at the different time moments. ${ }^{12}$ These subgroups were chosen based on practical considerations.

To assess whether the r_VLT-SF$_{100}$ allows the detection of improvement over time in different subgroups, the $\mathrm{r}_{-}$VLT-SF $F_{100}$ was statistically tested as to improvement using the Wilcoxon signed rank tests with a Bonferroni correction (alpha set at 0.025) for multiple comparisons between 2 different time intervals, that is, between $\mathrm{t} 1$ and $\mathrm{t} 2$ and between $\mathrm{t} 2$ and $\mathrm{t} 3$, for the total group and for the different subgroups.

\section{RESULTS}

Patient characteristics of the total group, the subgroups and time intervals are given in Table 1.

Table 2 presents the recoded VLT-SF raw scores, which were converted into $\mathrm{r}_{-} \mathrm{VLT}-\mathrm{SF}_{\text {logit }}$ scores using the Rasch analysis (as described in Spooren et al. ${ }^{4}$ ) and the transformation towards

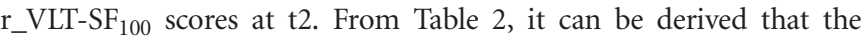
minimal recoded VLT-SF raw score of 0 corresponds to a r_VLT-SF score of -4.784 , which, in turn, matches with a r_VLT-SF 100 of 0.

\section{Table 2 Transformation of VLT-SF scores at $\mathrm{t} 2$}

$$
r_{-} V L T-S F
$$

\begin{tabular}{|c|c|c|c|}
\hline Raw score & Logits & s.e. & Transformed 0-100 score \\
\hline 0 & -4.784 & 1.27 & 0.0 \\
\hline 2 & -3.298 & 0.74 & 13.4 \\
\hline 4 & -2.475 & 0.60 & 20.8 \\
\hline 5 & -2.153 & 0.56 & 23.7 \\
\hline 6 & -1.864 & 0.54 & 26.3 \\
\hline 7 & -1.597 & 0.52 & 28.7 \\
\hline 8 & -1.345 & 0.50 & 31.0 \\
\hline 9 & -1.102 & 0.50 & 33.2 \\
\hline 10 & -0.866 & 0.49 & 35.3 \\
\hline 11 & -0.632 & 0.49 & 37.4 \\
\hline 12 & -0.397 & 0.49 & 39.5 \\
\hline 13 & -0.159 & 0.50 & 41.7 \\
\hline 14 & 0.085 & 0.50 & 43.9 \\
\hline 15 & 0.336 & 0.51 & 46.1 \\
\hline 16 & 0.597 & 0.52 & 48.5 \\
\hline 17 & 0.870 & 0.53 & 50.9 \\
\hline 18 & 1.159 & 0.55 & 53.5 \\
\hline 19 & 1.470 & 0.58 & 56.3 \\
\hline 20 & 1.812 & 0.61 & 59.4 \\
\hline 21 & 2.198 & 0.65 & 62.9 \\
\hline 22 & 2.645 & 0.70 & 66.9 \\
\hline 23 & 3.185 & 0.78 & 71.8 \\
\hline 24 & 3.877 & 0.91 & 78.0 \\
\hline 25 & 4.877 & 1.13 & 87.0 \\
\hline 26 & 6.317 & 1.54 & 100.0 \\
\hline
\end{tabular}

Abbreviations: s.e., standard error; VLT-SF, Van Lieshout hand function test for tetraplegia short form; r VLT-SF, Rasch-transformed Van Lieshout hand function test for tetraplegia short form.

Table 1 Patient characteristics and time intervals

\begin{tabular}{|c|c|c|c|}
\hline & $t 1$ & t2 & t3 \\
\hline$n$ & 61 & 73 & 69 \\
\hline High lesion (C1-C6) & $42(2 \mathrm{C} 3 ; 5 \mathrm{C} 4 ; 18 \mathrm{C} 5 ; 17 \mathrm{C} 6)$ & 45 (1C1; 2C4; 17C5; 25C6) & $34(1 \mathrm{C} 1 ; 1 \mathrm{C} 4 ; 14 \mathrm{C} 5 ; 18 \mathrm{C} 6)$ \\
\hline Low lesion (C7-T1) & 19 (10C7; 4C8; 5T1) & $28(14 \mathrm{C} 7 ; 6 \mathrm{C} 8 ; 8 \mathrm{~T} 1)$ & 35 (17C7; 7C8; 11T1) \\
\hline Motor complete (AB) & 33 & 35 & 31 \\
\hline Motor incomplete (CD) & 28 & 38 & 38 \\
\hline Gender (male/female) & $49 / 12$ & $53 / 20$ & $51 / 18$ \\
\hline Age (years)(mean)(s.d.) & $41(15)$ & $40(14,5)$ & $41(15)$ \\
\hline TSI (days)(mean)(s.d.) & $98(66)$ & $204(101)$ & $402(171)$ \\
\hline
\end{tabular}

Abbreviations: ASIA, American Spinal Injury Association; ISNCSCI, International Standard for Neurological Classification of Spinal Cord Injury; TSI, time since injury.

AB, Grade A or B on ASIA Impairment Scale; ${ }^{10} \mathrm{CD}$, Grade C or D on ASIA Impairment Scale; C, motor level according to the ISNCSCI. 

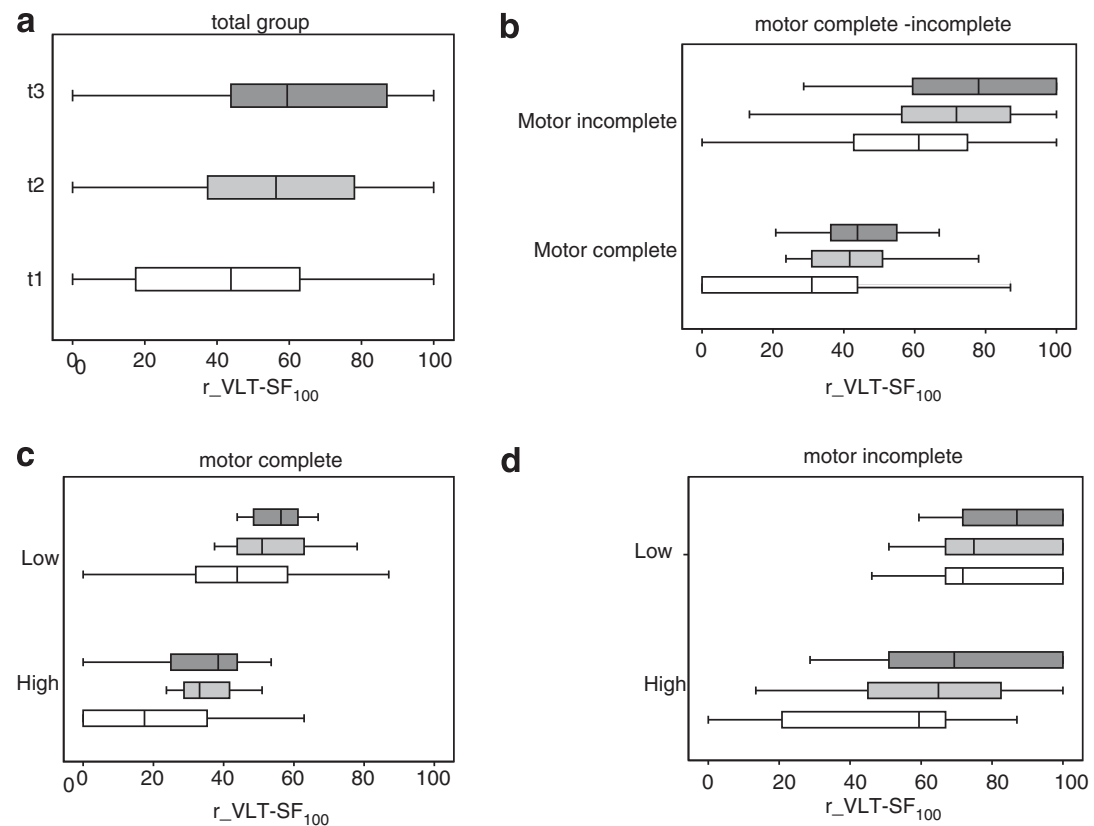

d

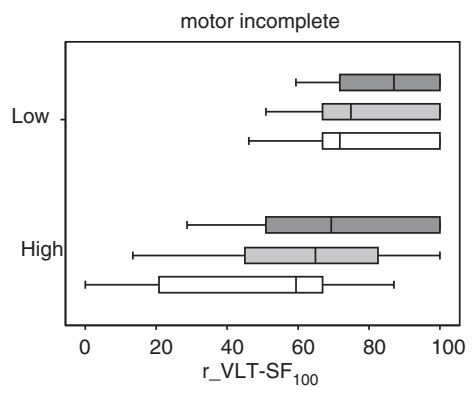

Figure 1 Reference values of $r_{-}$VLT-SF 100 in medians and interquartiles for different subgroups at $t 1, t 2$ and $t 3$. Reference values of $r$ VLT-SF $F_{100}$ at $t 1$, $t 2$ and $\mathrm{t} 3$ for different subgroups. $r_{-}$VLT-SF 100 (Rasch-tranformed VLT-SF expressed in $0-100$ scores). $\mathrm{t} 1=$ start of the active rehabilitation; $\mathrm{t} 2=3$ months after t1; t3 =discharge; (a) total group: t1 $(n=61)$, t2 $(n=73)$ and t3 $(n=69)$; (b) motor complete: t1 $(n=33)$, t2 $(n=38)$ and t3 $(n=35)$ and incomplete: t1 $(n=28)$, t2 $(n=35)$ and t3 $(n=34) ;(c)$ motor complete high (C7-T1) t1 $(n=21)$, t2 $(n=25)$ and t3 (n=23) and low (C1-C6): t1 $(n=12)$, t2 $(n=13)$ and t3 $(n=12)$ and; (d) motor incomplete high: t1 $(n=21)$, t2 $(n=26)$ and t3 $(n=26)$ and low t1 $(n=7)$, t2 $(n=9)$ and t3 $(n=8)$.

Table 3 Example of recoding of VLT-SF towards r_VLS-SF $_{(\text {raw })}$

\begin{tabular}{lllllll}
\hline Original VLT-SF response scale & 0 & 1 & 2 & 3 & 4 & 5 \\
Recoded VLT-SF response scale & & & & & & \\
$\quad$ Item 1: forward reaching & 0 & 1 & 1 & 2 & 2 & 3 \\
Item 2: arch task & 0 & 1 & 1 & 1 & 2 & 2 \\
Item 3 :opening thumb & 0 & 1 & 2 & 2 & 3 & 3 \\
Item 4: grip thumb & 0 & 1 & 1 & 2 & 2 & 3 \\
Item 5: strength thumb & 0 & 1 & 1 & 1 & 2 & 2 \\
Item 6: opening fingers & 0 & 1 & 1 & 1 & 2 & 2 \\
Item 7: strength fingers & 0 & 1 & 1 & 1 & 2 & 2 \\
Item 8: pen grip & 0 & 1 & 1 & 2 & 3 & 3 \\
Item 9: lighting match & 0 & 1 & 2 & 2 & 3 & 3 \\
Item 10: opening bottle & 0 & 1 & 2 & 2 & 3 & 3 \\
Total original VLT-SF & 7 & & & & & \\
Total r_VLT-SF(raw) & 6 & & & & & \\
r_VLT-SF(logit) & -1.86 & & & & & \\
r_VLT-SF(100) & 26 & & & & & \\
\hline
\end{tabular}

Abbreviations: VLT-SF, Van Lieshout hand function test for tetraplegia short form; r_VLT-SF raw, $_{\text {, }}$

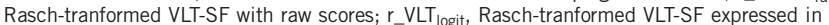
logits; r_VLT 100 , Rasch-tranformed VLT-SF expressed in 0-100 scores.

The maximal recoded VLT-SF raw score of 26 corresponds with a r_VLT-SF logit $_{\text {s }}$ score of 6.317 and with a r_VLT-SF 100 of 100 .

Based on these $r_{-}$VLT-SF 100 scores at t2, r_VLT-SF 100 scores were computed for $\mathrm{t} 1$ and $\mathrm{t} 3$ anchored on the scores at $\mathrm{t} 2$. Figure 1 depicts the reference values, that is, boxplots with median and interquartiles, for persons with motor complete and incomplete lesions with either high (C1-C6) or low (C7-T1) lesion level and for $\mathrm{t} 1$, $\mathrm{t} 2$ and $\mathrm{t} 3$.

Example: A tetraplegic patient with a motor complete lesion at C6 (AIS B and Sensor Level C5 according to ISNCSCI ${ }^{10}$ was admitted to rehabilitation. At the start of the rehabilitation, he obtained a score of
7 on the original VLT-SF (see Table 3). After recoding, the raw r_VLTSF score was 6 (see Table 2 and Table 3). From Table 2, it can be derived that the r_VLT-SF raw $_{\text {s }}$ score of 6 corresponds with a r_VLT$\mathrm{SF}_{\text {logit }}$ score of -1.86 . This score, in turn, corresponds with a r_VLT-SF 100 score of 26 (see Table 2). Based on the reference values at $\mathrm{t} 1$ for persons with a motor complete high lesion (see Figure 1c), it can be derived that he is performing quite well (above the median), and his expected outcome at discharge is estimated to be 40 .

A significant improvement $(P<0.025)$ was found not only between $\mathrm{t} 1$ and $\mathrm{t} 2$ and between $\mathrm{t} 2$ and $\mathrm{t} 3$ for the total group, but also for subgroups of persons with C-SCI (that is, for patients with a motor complete and incomplete lesion and for high and low lesion levels). A further analysis of subgroups revealed a significant improvement $(P<0.025)$ between $\mathrm{t} 1$ and $\mathrm{t} 2$ and between $\mathrm{t} 2$ and $\mathrm{t} 3$ for all the different subgroups (that is, for motor complete with either high or low lesion and motor incomplete with high lesion), except for the complete low lesion group between $\mathrm{t} 2$ and $\mathrm{t} 3(P=0.51)$ and for the incomplete low lesion groups between $\mathrm{t} 1$ and $\mathrm{t} 2(P=0.068)$ and $\mathrm{t} 2$ and $\mathrm{t} 3(P=0.109)$.

\section{DISCUSSION}

The aim of the present study was to (1) convert r_VLT-SF $_{\text {logit }}$ into $\mathrm{r}_{-}$VLT-SF ${ }_{100}$, (2) establish reference values of r_VLT-SF $_{100}$ for different groups of patients at different moments during rehabilitation and 3) verify whether the __VLT-SF $_{100}$ is is able to detect changes over time in different subgroups.

With regard to the conversion of scores, $r_{-}$VLT-SF $F_{\text {logit }}$ scores ranging from -4.784 to 6.317 logits were transformed into $\mathrm{r}_{-}$VLT-SF ${ }_{100}$ scores ranging from 0 to 100 . In clinical practice, this means that instead of reporting that a patient has a score of 0.87 logits on the r_VLT-SF ${ }_{\text {logit, }}$ it will be reported that he has a score of 51 on

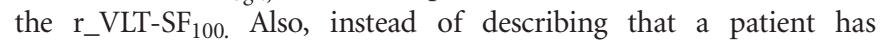


progressed from 0.87 to 3.19 logits, therapists can explain that the patient has progressed from 51 to 72 on the $r_{-}$VLT-SF $F_{100}$. As $0-100$ scores are more meaningful for clinicians and patients, the use of r_VLT-SF 100 scores will enhance communication between the former and the latter. Therefore, it is advisable to use the __VLT-SF $_{100}$ scores in clinical practice.

The most important aim of the present paper was to establish reference values regarding AHSP for different subgroups of patients with tetraplegia and for different stages in rehabilitation.

First, the r_VLT-SF 100 scores of $\mathrm{t} 1$ and $\mathrm{t} 3$ needed to be calculated. Theories on Rasch analysis indicate that one data set should be used as a primary set on which the data sets of other measurement moments should be anchored. ${ }^{5}$ In accordance with the former paper of Spooren et al., ${ }^{4}$ it was decided to take the $\mathrm{t} 2$ data as the primary data set. The $\mathrm{t} 2$ data set was considered for the majority of patients to be the most representative; that is, major floor or ceiling effects in patients who were either unable to perform any task (at the start of the rehabilitation) or patients who obtained the maximum score (at the end of the rehabilitation) were omitted. By anchoring the data of $\mathrm{t} 1$ and $\mathrm{t} 3$ to the $\mathrm{t} 2$ data set, it is possible to illustrate the progress on AHSP of an individual person during rehabilitation.

Reference values of AHSP of the r_VLT-SF ${ }_{100}$ for different groups of tetraplegia at different stages in the rehabilitation have been established by our research. Medians and interquartiles for the total group and different subgroups, that is, for persons with motor complete and incomplete lesions with either high (C1-C6) or low (C7-T1) lesion level at the start of the rehabilitation, 3 months after the start and at discharge, are now available.

The availability of reference values allows for a more accurate prediction of the outcome of AHSP. It provides more realistic (a-priori) information to the patient and may be used by clinicians who want to cross-reference their patients' performance regarding the therapy goals set and met. The use of reference values allows clinicians to take a more client-dedicated approach. First, it allows a more precise comparison between patients regarding AHSP at different time moments in order to answer the question 'Does my patient perform better or worse than patient $\mathrm{Y}$ at this particular stage?' Second, it enables referencing a patient's AHSP training outcome with standards obtained from the reference database to answer the question 'Is my patient's performance up to the standards (s)he should meet according to the norms?' In case the patient performs worse than required by the standards, the question rises, 'Why does (s)he perform worse?' Third, the clinician may relate the patient's performance to reference values to ascertain whether (and why) a final performance level has been reached (evaluation and explanation) or will be reached (prediction). The latter is also an important aspect for novice clinicians who have not (yet) acquired much 'hands-on' experience.

In clinical practice, the clinician may, in communicating with the patient, more clearly present realistic expectations and therapy results/ progress, and motivate and discuss any changes in therapy policy.

To verify that the r_VLT-SF is an instrument that allows the detection of improvement over time in different subgroups, the changes in the r_VLT-SF 100 outcome were statistically tested as to improvement. Based on the findings in the present study, in which statistically significant improvements in different stages during the rehabilitation and in most of the subgroups were found, it can be concluded that the r_VLT-SF 100 is able to detect changes over time in different subgroups. These findings corroborate earlier research findings by Spooren et al. ${ }^{11}$ on the original VLT-SF data, that is, that the VLT-SF is responsive to changes over time and that statistically significant improvements during rehabilitation were found in different subgroups. The lack of statistically significant differences in some subgroups and time intervals in the present study may be attributed to the small number of patients in, for example, the complete low lesion group $(n=12)$ and the incomplete low lesion group $(n=7)$.

The combination of good psychometric properties (criterion validity, the inter-rater reliability, the intra-rater reliability, the internal consistency and responsiveness), ${ }^{2,3}$ the application of the Rasch analysis ${ }^{4}$ and the establishment of reference values all contribute to the added value of the VLT in the management of upper extremity problems in tetraplegia. The VLT assesses the actual performance of arm and hand skills at the level of basic activities in persons with C-SCI and objectifies the strategy patients use to perform specific skills. It allows not only the assessment of the outcome and the progress of upper extremity performance but also the prediction of the outcome, and allows guiding therapists to plan progress in their therapy. In this way, the VLT is different from other instruments measuring upper extremity performances like the Capability Upper Extremity measure (CUE), ${ }^{13}$ the Tetraplegia Hand Activity Questionnaire (THAQ) ${ }^{14}$ or the Graded and Redefined Assessment of Strength, Sensibility and Prehension (GRASSP). The CUE is a self-reporting questionnaire in which patients rate their abilities to perform functional tasks with their arms; the THAQ focuses on activities that are important to persons with SCI, ${ }^{15}$ but has limited clinical application being reported. ${ }^{14}$ The recently developed GRASSP is a very promising instrument, designed to track the extent of spontaneous recovery or possible outcomes of a surgical or pharmacological intervention ${ }^{16,17}$ and combines the evaluation of the impairment level with the evaluation of prehension. In contrast to the VLT, the GRASPP does not include information on the arm performance and it cannot be used as both an assessment tool and therapy guideline. Furthermore, psychometric properties regarding responsiveness still need to be reported.

Although reference values of the VLT have been established in the present study, some considerations should be made. As the sample size of some subgroups is rather small, some caution in interpreting the reference values of these subgroups is warranted. It would be ideal to have reference values according to the completeness of the lesion for each lesion level. However, the data set is still limited. The data set is planned to be expanded in order to increase the accuracy of prediction and to be able to make more refined inferences leading to more explicit conclusions. Also, the present paper demonstrated the reference values of the best hand; that is, the VLT scores of the best hand were taken. As the performance of both hands may differ, results of both hands would give more precise information with regard to prediction and comparison.

To conclude, the r_VLT-SF logit $_{\text {s }}$ scores were converted into r_VLT$\mathrm{SF}_{100}$ scores, which may facilitate communication amongst clinicians and patients. The reference values of the $\mathrm{r}_{-}$VLT-SF ${ }_{100}$ have been established for different subgroups of patients with tetraplegia at different stages of rehabilitation and $\mathrm{r}_{-}$VLT-SF 100 has been proven to be able to detect improvement over time. The availability of reference values may improve the evaluation, prediction, rehabilitation management and decision making regarding AHSP.

\section{DATA ARCHIVING}

There were no data to deposit.

\section{CONFLICT OF INTEREST}

The authors declare no conflict of interest. 


\section{ACKNOWLEDGEMENTS}

This study was supported by ZonMW (grant no. 335020023).

1 Post MWM. Pilot-onderzoek Van Lieshout Test (in Dutch). Hoensbroek: iRv 2000.

2 Post MW, Van Lieshout G, Seelen HA, Snoek GJ, Ijzerman MJ, Pons C. Measurement properties of the short version of the Van Lieshout test for arm/hand function of persons with tetraplegia after spinal cord injury. Spinal Cord 2006; 44: 763-771.

3 Spooren AI, Janssen-Potten YJ, Post MW, Kerckhofs E, Nene A, Seelen HA. Measuring change in arm hand skilled performance in persons with a cervical spinal cord injury: responsiveness of the Van Lieshout test. Spinal Cord 2006; 44: 772-779.

4 Spooren AIF, Arnould C, Smeets RJEM, Bongers HMH, Seelen HAM. Improvement of the Van Lieshout hand function test for Tetraplegia using a Rasch analysis. Spinal Cord 2013; 51: 739-744.

5 Bond TG, Fox CM. Applying the Rasch model: Fundamental Measurement in the Human Sciences, 2nd edn. Taylor \& Francis group: Abingdon: Routledge, Oxford, UK, 2007.

6 Bouffioulx E, Arnould C, Thonnard JL. Satisfaction with activity and participation and its relationships with body functions, activities, or environmental factors in stroke patients. Arch Phys Med Rehabil 2011; 92: 1404-1410.

7 Arnould C, Penta M, Renders A, Thonnard JL. ABILHAND-Kids: a measure of manual ability in children with cerebral palsy. Neurology 2004: 63: 1045-1052.

8 Spooren AIF, Janssen-Potten YJM, Kerckhofs E, Seelen HAM. ToCUEST: a taskoriented client-centred training module to improve upper extremity skilled performance in cervical spinal cord injured persons. Spinal Cord 2011; 10: 1042-1048.
9 Spooren AIF, Janssen-Potten YJM, Kerckhofs E, Seelen HAM. Evaluation of a taskoriented client-centred training program to improve arm hand skilled performance in cervical spinal cord injury. Spinal Cord 2011; 10: 1049-1054.

10 Kirshblum SC, Burns SP, Biering-Sorensen F, Donovan W, Graves DE, Jha A et al. International standards for neurological classification of spinal cord injury (revised 2011). J Spinal Cord Med 2011; 34: 535-546.

11 Spooren AIF, Janssen-Potten YJM, Snoek GJ, Ijzerman MJ, Kerckhofs E, Seelen H. Rehabilitation outcome of upper extremity skilled performance in persons wiht cervical spinal cord injuries. J Rehab Med 2008; 40: 637-644.

12 Harvey L, Simpson D, Glinksy J, Pirronello D, McLean S. Quantifying the passive extensibility of the flexor pollicis longus mulscle in peple wiht tetrplegia. Spinal Cord 2005; 43: 620-624.

13 Marino RJ, Shea JA, Stineman MG. The capabilities of upper extremity instrument: reliability and validity of a measure of functional limitation in tetraplegia. Arch Phys Med Rehabil 1998; 79: 1512-1521.

14 Mulcahey MJ, Hutchinson D, Kozin S. Assessment of upper limb in tetraplegia: considerations in evaluation and outcomes research. J Rehabil Res Dev 2007; 44: 91-102.

15 Snoek GJ, IJzerman MJ, Hermens HJ, Maxwell D, Biering-Sorensen F. Survey of the needs of patients with spinal cord injury: impact and priority for improvement in hand function in tetraplegics. Spinal Cord 2004; 42: 526-532.

16 Alexander MS, Anderson KD, Biering-Sorensen F, Blight AR, Brannon R, Bryce TN et al. Outcome measures in spinal cord injury: recent assessments and recommendations for future directions. Spinal Cord 2009; 47: 582-591.

17 Kalsi-Ryan S, Curt A, Verrier MC, Fehlings MG. Development of the Graded Redefined Assessment of Strength, Sensibility and Prehension (GRASSP): reviewing measurement specific to the upper limb in tetraplegia. J Neurosurg Spine 2012; 17 (Suppl 1), 65-76. 